\title{
TWO NEW DECISION DIRECTED M-PSK TIMING ERROR DETECTORS
}

\author{
Yair Linn \\ University of British Columbia \\ 2111 Lower Mall, Vancouver, BC, Canada, V6T-1Z4 \\ e-mail: ylinn@ece.ubc.ca
}

\begin{abstract}
This paper shall introduce two new Decision Directed timing error detectors for symbol synchronization circuits in M-PSK receivers. The first detector is a modification of the Gardner timing error detector and requires a sampling rate of 2 samples/symbol. The second detector is a modification of the Mueller \& Müller detector and requires 1 sample/symbol. The analysis of the detectors will encompass 1) computation of their S-curves, 2) computation of their tracking gains, 3) discussion of their hardware implementation, 4) evaluation of their timingerror variance, and, finally, 5) comparison of the detectors to the unmodified versions and to other timing error detectors, especially with regards to fading and resistance to Automatic Gain Control circuit imperfections.
\end{abstract}

Keywords - symbol, synchronization, timing, error, detector.

\section{Introduction}

\subsection{Overview of Paper}

Every M-PSK (M-ary Phase Shift Keying) receiver includes a symbol synchronization circuit. The purpose of this circuit, which in general is a Phase Locked Loop (PLL), is to determine the optimal sampling instances of the $I$ and $Q$ channels so that the Symbol Error Rate (SER) is minimized. The PLL does this by attempting to nullify an estimate of the timing error that exists between the local and received symbol clocks.

The timing error estimate is provided to the PLL by a Timing Error Detector (TED). One such TED is the Gardner detector[2], which requires a sampling rate of 2 samples/symbol and has both Non Data Aided (NDA) and Decision Directed (DD) versions. Another popular TED is the Mueller \& Müller (M\&M) detector[1], which requires 1 sample/symbol and is a DD detector.

In this paper we present two new Decision Directed TEDs. The first, which requires 2 samples/symbol, is a modification of the Decision Directed Gardner detector.
The second, which requires 1 sample/symbol, is a modification of the Mueller \& Müller detector. The analysis of the proposed detectors will begin with the computation of their S-curves. Then, the new detectors will be shown to be more robust than the originals with regards to resilience to fading and Automatic Gain Control (AGC) circuit imperfections. Additionally, the performance of the proposed detectors will be evaluated through simulations, which will prove that the new detectors have timing error variance performance which is at least as good as that of the original detectors. Moreover, it shall be shown that the new detectors allow for a reduction in the dynamic range that the symbol synchronization PLL needs to handle, hence simplifying the receiver's implementation. Finally, the new detectors will be shown to have a simple hardware structure that is ideal for use within a Field Programmable Gate Array (FPGA) or an Application Specific Integrated Circuit (ASIC).

We shall first deal with BPSK and QPSK. At the end of the paper we shall generalize the results for higher order modulations.

\subsection{Definition of Acronyms for Detector Names}

Let us now take a short detour in order to define some notations which shall help facilitate some brevity of discourse. First, regarding the Gardner detectors: in this paper we write GNDA to refer to the Gardner NDA TED, and GDD to refer to the Gardner DD TED. The proposed detector, as shall be seen, can be thought of as a GDD which undergoes adaptive normalization. Hence, we use the notation N-GDD to refer to the modified detector.

In the professional literature the Mueller \& Müller detector is often referred to, somewhat affectionately, as the M\&M (pronunciation: M-N-M) detector. This convention will be adopted here as well. Since the second proposed detector is obtained through normalization of the M\&M, with equal affection we christen the new detector N-M\&M (pronunciation: N-M-N-M), short for "Normalized Mueller \& Müller". 


\subsection{Context of the Proposed Detectors}

Since the proposed detectors are modifications of the GDD and M\&M detectors, in this paper the GDD and $M \& M$ detectors will be the primary detectors opposite which the N-GDD and N-M\&M will be compared. That having been said, a brief overview of some other detectors will now be given, so as to delineate the place that the N-GDD and N-M\&M inhabit within the realm of contemporarily available detectors.

The GNDA, GDD and M\&M suffer from the inherent drawback that their S-curves are dependent upon the AGC's operating point and performance. As has already been noted, one of the greatest advantages of the N-GDD and N-M\&M is that they are AGC independent. Regarding the GNDA, a modified detector which remedies the AGC-dependence problem has been introduced in [9]; indeed, the N-GDD can be though of as the Decision Directed counterpart of the detector in [9].

Perhaps the most fetching advantage of the M\&M detector - and which the N-M\&M detector retains - is that fact that it necessitates only 1 sample/symbol. The Gardner detectors, in contrast, have the disadvantage of requiring 2 samples/symbol. One of the disadvantages of the M\&M, though, is that in order to function properly it requires prior carrier synchronization. This limitation has been found, through simulations, to exist regarding the $\mathrm{N}$ $\mathrm{M} \& \mathrm{M}$ as well. Hence, the analysis in this paper which concerns the M\&M and N-M\&M assumes that the local carrier is coherent with the received one. A carrierindependent modification of the M\&M TED was proposed in [6] (the modified detector being referred to there as the DD-1M detector). The detector proposed in [6] tackles the carrier phase error problem by performing coarse carrier phase correction of the $I$ and $Q$ channels before the M\&M algorithm is applied. It would be interesting to try and apply the same procedure with regards to the N-M\&M, though this is beyond the scope of the current paper. There have also been algorithms suggested in [7] which provide a timing error estimate that is independent of the carrier phase, at the sampling rate of 1 sample/symbol. However it must be stressed that the constructs in [6] and [7] do not have the AGCindependence which the N-M\&M possesses.

When timing synchronization is needed even without prior carrier synchronization, one often chooses to use the GNDA or GDD, which, as shown in [2], can operate even when the carrier loop is unlocked (the introduction of [2] also contains a good overview of various TEDs which will not be discussed here). The N-GDD, as simulations conducted by the author have shown, retains this capability to operate even in the absence of carrier synchronization, as does the detector in [9]. Other carrierindependent detectors operating at 2 samples/symbol have

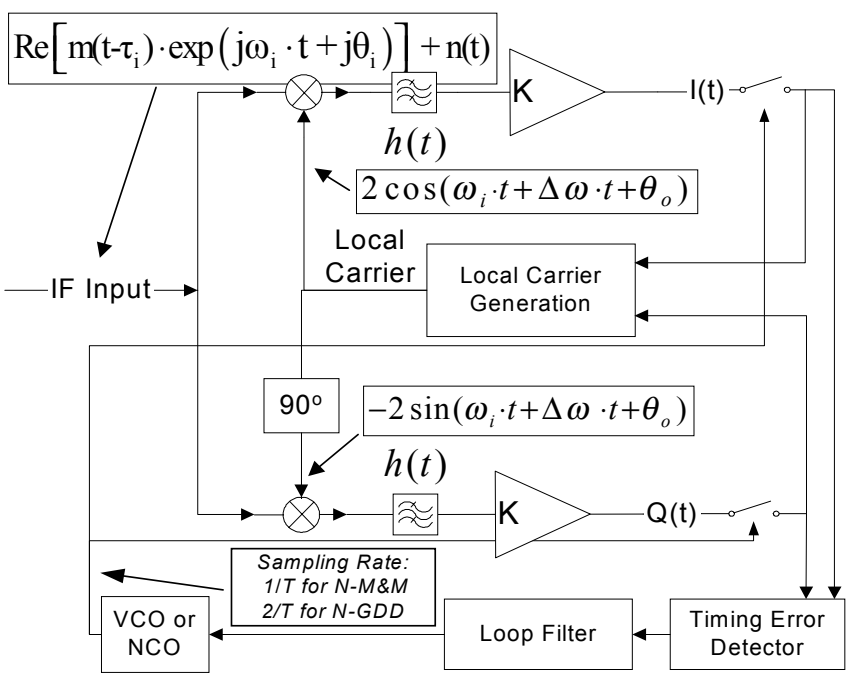

Fig. 1. Simplified M-PSK receiver structure.

also been suggested in [8] and [11], though neither possess the AGC-independence of the N-GDD.

\section{Signal Definitions and Receiver Structure}

Denote the baseband pulse shape as $p(t)$ and the baseband signal

as $m(t)=\sum_{r=-\infty}^{\infty}\left(a_{r} / \sqrt{2}+j \cdot b_{r} / \sqrt{2}\right) p(t-r T), \quad$ with $1 / T$ being the symbol rate. For BPSK $a_{r} \in\{-\sqrt{2}, \sqrt{2}\}$ and $b_{r}=0$ for all $r$, while in the case of QPSK we have $a_{r}, b_{r} \in\{-1,1\}$. We use $\tau_{i}$ to signify the propagation delay, and the received signal is then $s_{m}(t)=\operatorname{Re}\left[m\left(t-\tau_{i}\right) \exp \left(j \omega_{i} t+j \theta_{i}\right)\right]$, which is corrupted by AWGN (Additive White Gaussian Noise). The structure of the receiver is shown in Fig. 1, where:

1. $n(t) \sim N\left(0, N_{0} W\right)$ where $W$ is the width of the bandpass IF filter before the $I-Q$ demodulator (not shown).

2. Unless otherwise stated, we assume a local carrier which is coherent with the received carrier (i.e. $\Delta \omega=0$ and $\left.\theta_{i}=\theta_{o}\right)$. The local carrier may be generated via such methods as a Costas-type PLL ([3],[10]), a pilotaided carrier regeneration scheme, or other methods.

3. The matched filters $h(t)=p(-t)$ are assumed ideal.

4. $K$ represents the equivalent physical gain of the $I$ and $Q$ arms. For simplicity, $K$ is assumed the same in both those arms, though this is not a necessary condition for the analysis presented here regarding the N-GDD and N-M\&M (this is, however, a necessary condition for both the GDD and M\&M to perform as expected for $M>2$ ). 
$K$ is a slowly time-variant function of the $\mathrm{AGC}$, which attempts to control the signal amplitude levels at the inputs of the samplers so that the full dynamic range of the samplers is utilized yet the samplers do not reach saturation. The N-GDD and N-M\&M will be found to be independent of $K$, which is their defining advantage vs. the original detectors, since this means that a symbol timing synchronization PLL which uses the normalized detectors will be decoupled from the AGC circuit's operating point and performance. These issues are elaborated upon in the remainder of the paper.

In this paper we shall assume that the baseband pulse is of the Square-Root Raised-Cosine (SRRC) type, that is ([4] eq. 68.15):

$$
p(t)=4 \alpha \frac{\cos ((1+\alpha) \pi t / T)+\frac{\sin ((1-\alpha) \pi t / T)}{4 \alpha t / T}}{\pi \sqrt{T}\left(1-16 \alpha^{2} t^{2} / T^{2}\right)}
$$

which yields the well-known raised-cosine channel response of ([3] eq. 2.2.10)

$$
g(t) \triangleq p(t) \otimes h(t)=\frac{\sin (\pi t / T)}{\pi t / T} \cdot \frac{\cos (\pi \alpha t / T)}{1-4 \alpha^{2} t^{2} / T^{2}} .
$$

The constant $0 \leq \alpha \leq 1$ is the "rolloff" factor.

\section{N-GDD Detector Definition}

$$
\begin{aligned}
& \text { We have in Fig. } \\
& I(t)=K\left(\sum_{r=-\infty}^{\infty} \frac{a_{r}}{\sqrt{2}} g\left(t-r T-\tau_{i}\right)+n_{I}(t)\right) \\
& Q(t)=K\left(\sum_{r=-\infty}^{\infty} \frac{b_{r}}{\sqrt{2}} g\left(t-r T-\tau_{i}\right)+n_{Q}(t)\right) \\
& n_{I}(t), n_{Q}(t) \sim N\left(0, N_{0} \int_{-\infty}^{\infty} h^{2}(t) d t\right) \text {. For the N-GDD (as }
\end{aligned}
$$

$$
\text { shown in Fig. 1), the sampling rate is } 1 / T_{S}=2 / T \text {. We }
$$
use $\hat{\tau}_{i}$ to refer to the receiver's estimate of $\tau_{i}$, and we denote the even samples of the channels as:

$$
\left.\left.I_{e}(n) \triangleq I(t)\right|_{t=2 n T_{S}+\hat{\tau}_{i}} Q_{e}(n) \triangleq Q(t)\right|_{t=2 n T_{S}+\hat{\tau}_{i}}
$$

and the odd samples as:

$$
\left.\left.I_{o}(n) \triangleq I(t)\right|_{t=(2 n+1) T_{S}+\hat{\tau}_{i}} \quad Q_{o}(n) \triangleq Q(t)\right|_{t=(2 n+1) T_{S}+\hat{\tau}_{i}}
$$

The symbol synchronization timing error is defined as $\tau \triangleq\left(\hat{\tau}_{i}-\tau_{i}\right) \bmod _{T}$, where the modulo operation yields $\tau \in[-T / 2, T / 2]$. We further define:

$$
\begin{aligned}
& v_{I}(n) \triangleq \operatorname{sign}\left(I_{e}(n+1)\right) \frac{I_{o}(n)}{\sqrt{I_{e}^{2}(n+1)+I_{o}^{2}(n)}} \\
& -\operatorname{sign}\left(I_{e}(n)\right) \frac{I_{o}(n)}{\sqrt{I_{e}^{2}(n)+I_{o}^{2}(n)}}
\end{aligned}
$$

$$
\begin{aligned}
& v_{Q}(n) \triangleq \operatorname{sign}\left(Q_{e}(n+1)\right) \frac{Q_{o}(n)}{\sqrt{Q_{e}{ }^{2}(n+1)+Q_{o}{ }^{2}(n)}} \\
& -\operatorname{sign}\left(Q_{e}(n)\right) \frac{Q_{o}(n)}{\sqrt{Q_{e}{ }^{2}(n)+Q_{o}{ }^{2}(n)}}
\end{aligned}
$$

We now define the N-GDD using (5)-(6). For QPSK, the N-GDD is:

$$
v(n) \triangleq v_{I}(n)+v_{Q}(n)
$$

As for BPSK, in the absence of carrier synchronization the detector is also (7). When carrier lock is attained the $Q$ arm contains only noise, and then the N-GDD for BPSK is $v_{I}(n)$, given in (5). It is worth noting that if the denominator terms are omitted in $v_{I}(n)$ and $v_{Q}(n)$, one is left with the GDD ([2],[3]) which for QPSK (or unlocked carrier and BPSK) is written as $I_{o}(n)\left(\operatorname{sign}\left(I_{e}(n+1)\right)-\operatorname{sign}\left(I_{e}(n)\right)\right)$ $+Q_{o}(n)\left(\operatorname{sign}\left(Q_{e}(n+1)\right)-\operatorname{sign}\left(Q_{e}(n)\right)\right)$, and for BPSK with a locked carrier loop is given by $I_{o}(n)\left(\operatorname{sign}\left(I_{e}(n+1)\right)-\operatorname{sign}\left(I_{e}(n)\right)\right)$. The function of the denominator terms of $v_{I}(n)$ and $v_{Q}(n)$ is the performance of adaptive normalization of the TED, an action that strongly influences the detector's characteristics. In particular, the normalization eliminates any dependence of the detector on $K$, a fact which is demonstrated if one inserts the expressions for $I(t)$ and $Q(t)$ into (3) through (7). In contrast, it is equally trivial to conclude, from inspection, that the GDD's output is proportional to $K$ (because $\left.I_{o}(n), Q_{o}(n) \propto K\right)$. The dependence of the GDD on $K$ means that the GDD's gain will be affected by any uncountered non-ideal operation of the AGC loop (i.e. an AGC that produces non-nominal values of $K$ ). Such variations in $K$ will also change the symbol synchronization PLL's loop gain, damping factor and loop bandwidth (see [5] chap. 9). Similar phenomena can be easily shown to afflict the GNDA as well[9]. Again, since $v_{I}(n), v_{Q}(n)$ and $v(n)$ are independent of $K$, they are thus immune to such effects. This is of special importance when fading signals are encountered, a scenario in which the AGC often exhibits distinctly non-ideal behaviour.

\section{N-M\&M Detector Definition}

For the N-M\&M (as shown in Fig. 1), the sampling rate is equal to the symbol rate $1 / T$, and thus the 
samples of the channels are $\left.I(n) \triangleq I(t)\right|_{t=n T+\hat{\tau}_{i}}$ and $\left.Q(n) \triangleq Q(t)\right|_{t=n T+\hat{t}_{i}}$. We define:

$e_{I}(n) \triangleq \frac{\operatorname{sign}(I(n-1)) I(n)-\operatorname{sign}(I(n)) I(n-1)}{\sqrt{I^{2}(n-1)+I^{2}(n)}}$

$e_{Q}(n) \triangleq \frac{\operatorname{sign}(Q(n-1)) Q(n)-\operatorname{sign}(Q(n)) Q(n-1)}{\sqrt{Q^{2}(n-1)+Q^{2}(n)}}$

For the case of BPSK the N-M\&M is $e_{I}(n)$, given in (8). For QPSK, the N-M\&M is:

$e(n) \triangleq e_{I}(n)+e_{Q}(n)$.

Observe that if the denominator terms are omitted from $e_{I}(n)$ and $e_{Q}(n)$, one is left with the M\&M ([1],[3]) detector, which for QPSK is written as

$(\operatorname{sign}(I(n-1)) I(n)-\operatorname{sign}(I(n)) I(n-1))$
$+(\operatorname{sign}(Q(n-1)) Q(n)-\operatorname{sign}(Q(n)) Q(n-1))$
for and
$\operatorname{sigh}(I(n-1) I(n)-\operatorname{sign}(I(n)) I(n-1)$ $\operatorname{sign}(I(n-1)) I(n)-\operatorname{sign}(I(n)) I(n-1)$.

The denominator terms of $e_{I}(n)$ and $e_{Q}(n)$ can be thought of as performing adaptive normalization of the M\&M. In particular (just as was the case with the NGDD) the normalization removes any dependence of the N-M\&M on $K$, as can be seen if one inserts the expressions for $I(t)$ and $Q(t)$ into (8) through (10). The same benefits vis-à-vis the $\mathrm{AGC}$ will be observed here as were observed for the N-GDD.

\section{S-curves}

The S-curve of a given detector $d(n)$ is computed via $S_{d}(\tau) \triangleq E[d(n) \mid \tau]$. The gain of the detector is defined as the magnitude of the slope of the S-curve at $\tau=0$. We present S-curves for the N-GDD and the NM\&M obtained through computer simulations, in Fig. 2Fig. 4. It is important to emphasize that the results for the GDD and M\&M in those figures assume a time-invariant and constant $K=1$, while this assumption is not needed for the data for the N-GDD or N-M\&M. In particular, if $K \neq 1$ the gains and curves for the GDD and M\&M will scale as a function of $K$, whereas those for the N-GDD and N-M\&M would remain unchanged.

\section{Performance Evaluation}

To quantitatively assess the detectors' performance, we look at the variance of the normalized timing error during tracking as obtained through closed-loop simulations of a baseband equivalent model of Fig. 1 .
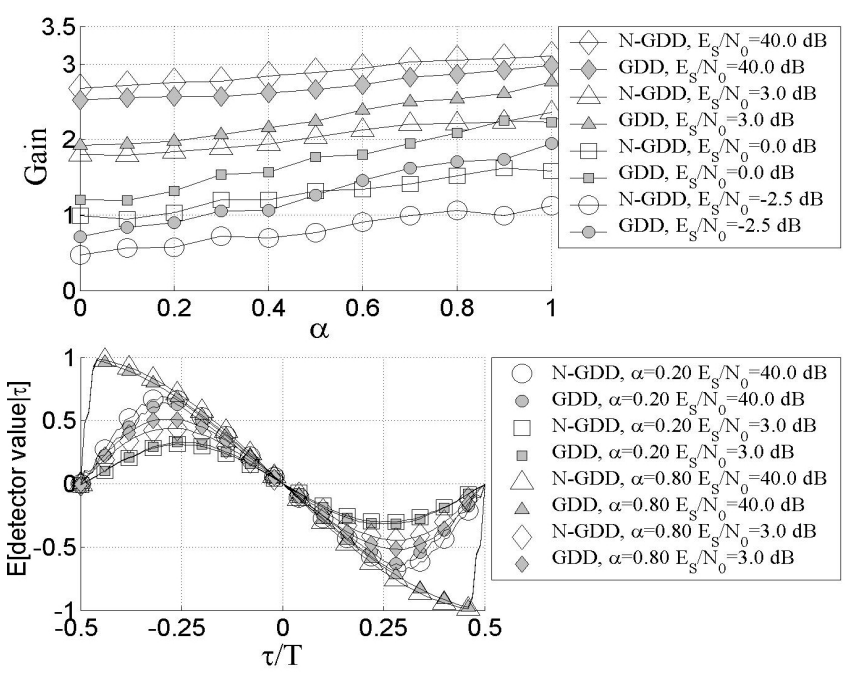

Fig. 2. S-curves, BPSK input signals. Top shows the detector gains, bottom shows sample S-curves.
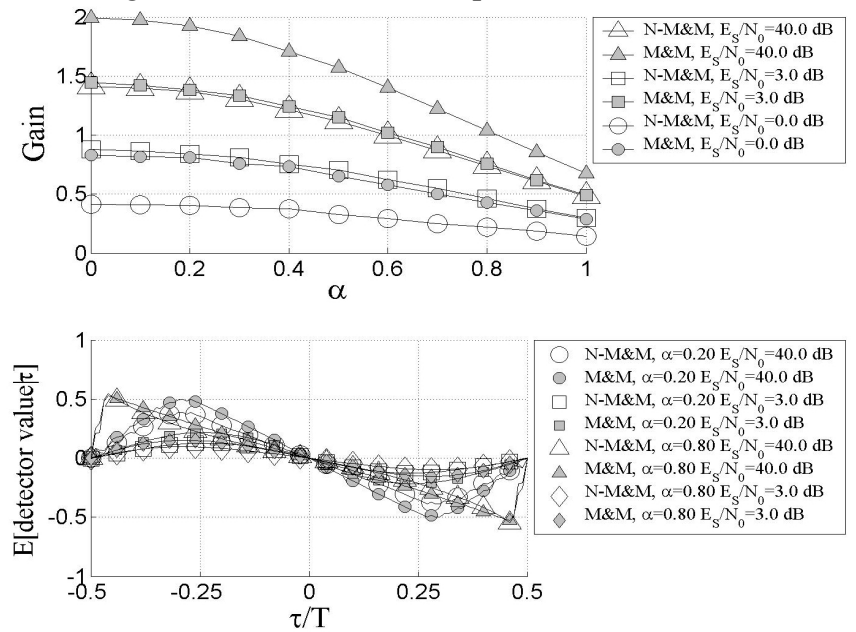

Fig. 3. S-curves, BPSK input signals. Top shows the detector gains, bottom shows sample S-curves.
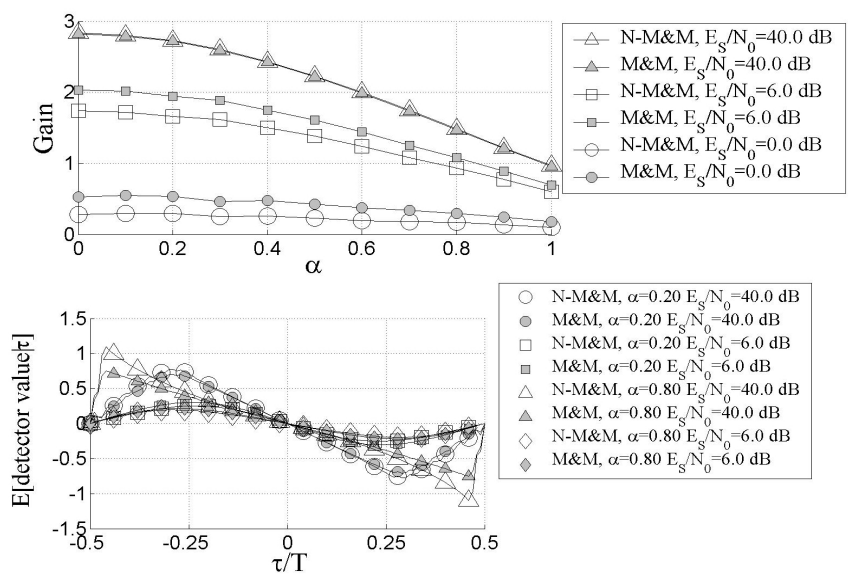

Fig. 4. S-curve, QPSK input signals. Top shows the detector gains, bottom shows sample S-curves. 
Results are shown in Fig. 5 to Fig. 7, for $\alpha=0.25$ and $\alpha=0.75$, for a normalized synchronization loop noise bandwidth[3] of $B_{L} T=5 \cdot 10^{-3}$. Also plotted in those figures (for comparison) are variances obtained when the GDD, GNDA, and M\&M detectors were used, as well as the Modified Cramér-Rao Bounds (MCRB), given by ([3] eqs. 7.4.55 and 7.4.78):

$M C R B=\frac{B_{L} T}{4 \pi^{2} \xi} \cdot \frac{1}{E_{S} / N_{0}}$,

$\xi=\frac{1}{4}\left(\frac{1}{3}+\alpha^{2}\left(1-\frac{8}{\pi^{2}}\right)\right)$.

As seen from Fig. 5 and Fig. 6, the N-GDD usually performs better than the GNDA detector (particularly for a small rolloff coefficient, as well as at high-SNRs), and performs virtually identically as compared to the GDD.

Similarly, regarding the N-M\&M, Fig. 7 shows that the N-M\&M performs similarly vs. the M\&M (slightly worse than the M\&M at low SNR). Results for QPSK are almost identical and are thus omitted.

As an additional verification of the results of Fig. 5 and Fig. 7, observe that the results for the M\&M, GDD, and GNDA agree well with those achieved in [3] using similar system parameters (see [3] Figs. 7.20, 7.21, 7.22, and 7.23, where in [3] MMD denotes the M\&M detector, $\mathrm{ZCD}$ is used to denote the GDD, and GAD is used to denote the GNDA).

Finally, one must remember that the results for the M\&M, GDD, and GNDA in Fig. 5 through Fig. 7 assume a known and constant $K$, a requirement which, in contrast, is not necessary neither for the N-GDD nor for the N-M\&M. Put another way, there is an extra degree of robustness that the N-GDD and N-M\&M possess and which is not directly reflected in Fig. 5-Fig. 7.

\section{Theoretical Motivation}

There is straightforward reasoning which underlies the motivation for synthesizing the N-GDD and N-M\&M. Dealing first with the N-GDD and for simplicity assuming a BPSK signal and a locked carrier, let us study $v_{I}(n)$ as expressed in (5). If we define $\varphi_{n}^{-}=\tan ^{-1}\left(I_{o}(n) / I_{e}(n)\right)$ and $\varphi_{n}^{+}=\tan ^{-1}\left(I_{o}(n) / I_{e}(n+1)\right)$ then it is easy to show that

$$
\begin{aligned}
& v_{I}(n)=\operatorname{sign}\left(I_{e}(n+1)\right) \sin \left(\varphi_{n}^{+}\right) \\
& -\operatorname{sign}\left(I_{e}(n)\right) \sin \left(\varphi_{n}^{-}\right)
\end{aligned}
$$

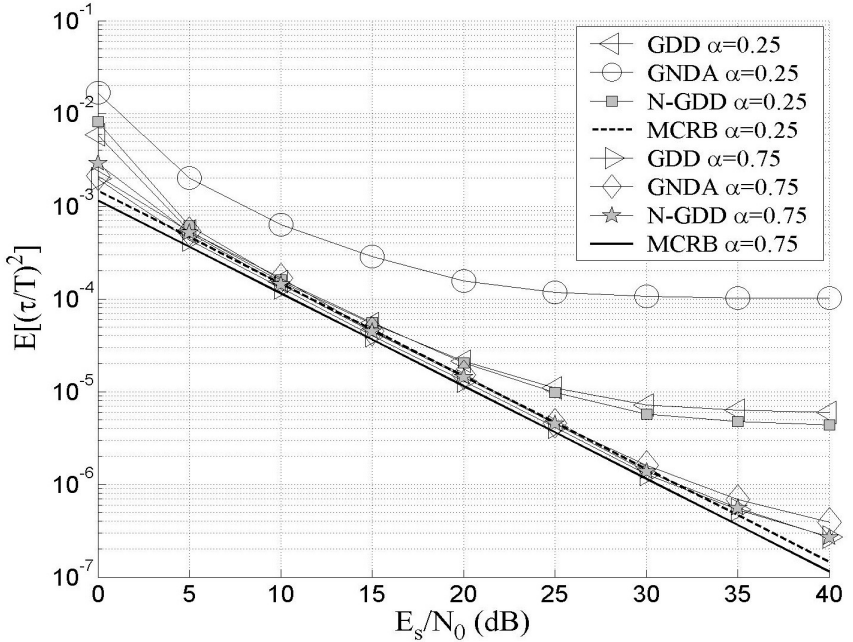

Fig. 5. Normalized timing error variance, BPSK, for NGDD, GDD, and GNDA, with $B_{L} T=5 \cdot 10^{-3}$.

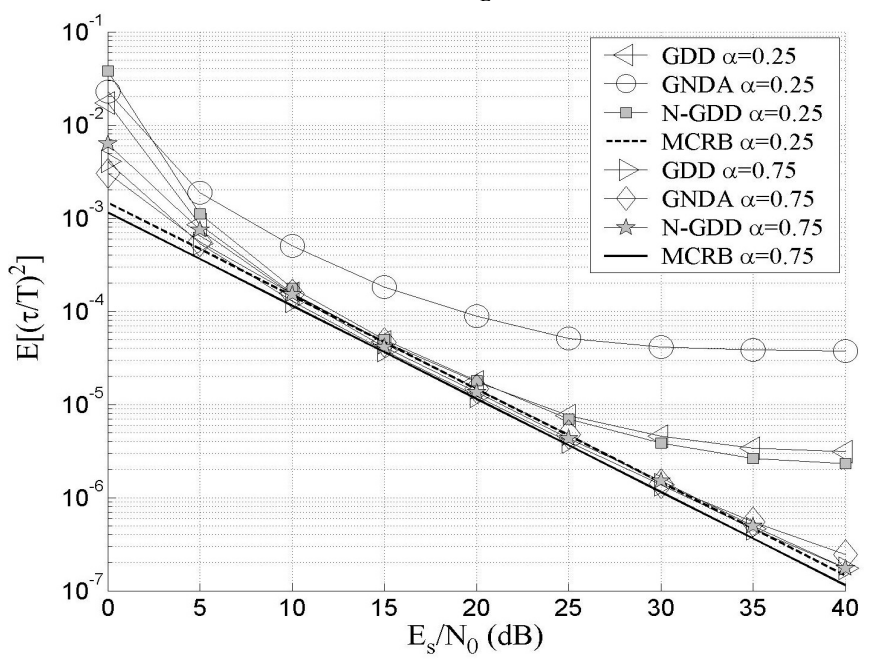

Fig. 6 Normalized timing error variance, QPSK, for NGDD, GDD, and GNDA, with $B_{L} T=5 \cdot 10^{-3}$.

$$
\begin{aligned}
& G D D_{I}(n)=\operatorname{sign}\left(I_{e}(n+1)\right) \sin \left(\varphi_{n}^{+}\right) \sqrt{I_{e}^{2}(n+1)+I_{o}^{2}(n)} \\
& -\operatorname{sign}\left(I_{e}(n)\right) \sin \left(\varphi_{n}^{-}\right) \sqrt{I_{e}^{2}(n)+I_{o}^{2}(n)} \\
& \text { The factors } \quad \sqrt{I_{e}^{2}(n+1)+I_{o}^{2}(n)}
\end{aligned}
$$
and $\sqrt{I_{e}^{2}(n)+I_{o}^{2}(n)}$ are proportional to $K$ and are a function of the total power of the received signal (including noise). The signal's power does not contribute timing information, and so it is no surprise that by eliminating the error signal's dependence on those factors the performance of the detector is almost unchanged vs. that of the GDD.

Similarly for the N-M\&M (again looking at BPSK and a locked carrier) if we define: 


$$
\begin{aligned}
& \varphi_{n} \triangleq \tan ^{-1}(I(n-1) / I(n)) \\
& =\tan ^{-1}\left(\frac{\sum_{r=-\infty}^{\infty} \frac{a_{r}}{\sqrt{2}} g((n-1) T-r T+\tau)+n_{I}\left((n-1) T+\hat{\tau}_{i}\right)}{\sum_{r=-\infty}^{\infty} \frac{a_{r}}{\sqrt{2}} g(n T-r T+\tau)+n_{I}\left(n T+\hat{\tau}_{i}\right)}\right)(1
\end{aligned}
$$

then we have that the N-M\&M's signal is:

$$
e_{I}(n) \triangleq \frac{\sqrt{I^{2}(n-1)+I^{2}(n)}\left(\begin{array}{c}
\operatorname{sign}(I(n-1)) \cos \left(\varphi_{n}\right) \\
-\operatorname{sign}(I(n)) \sin \left(\varphi_{n}\right)
\end{array}\right)}{\sqrt{I^{2}(n-1)+I^{2}(n)}}
$$$$
=\operatorname{sign}(I(n-1)) \cos \left(\varphi_{n}\right)-\operatorname{sign}(I(n)) \sin \left(\varphi_{n}\right)
$$

whereas the M\&M's error signal would be:

$\operatorname{sign}(I(n-1)) I(n)-\operatorname{sign}(I(n)) I(n-1)$

$$
=\sqrt{I^{2}(n-1)+I^{2}(n)}\left(\begin{array}{c}
\operatorname{sign}(I(n-1)) \cos \left(\varphi_{n}\right) \\
-\operatorname{sign}(I(n)) \sin \left(\varphi_{n}\right)
\end{array}\right)
$$

Though closed-form theoretical justification of the numerical results obtained in previous sections appears unattainable due to the infinite sums in (14), the NM\&M's behaviour can be understood both through both heuristic and graphical methods. First, as a heuristic explanation, we see from (15)-(16) that the N-M\&M (as compared to the M\&M) eliminates the multiplicative dependency of the error signal upon the quantity $\sqrt{I^{2}(n-1)+I^{2}(n)}$, which is proportional to $K$ and is a function of the total power of the received signal (including noise). As already noted, the signal's power does not contribute timing information, so it is no surprise that by eliminating the error signal's dependency on $\sqrt{I^{2}(n-1)+I^{2}(n)}$ we achieve such benefits as a resilience to AGC imperfections and better operation in fading conditions, while at the same time having virtually no impact on the timing error variance performance of the timing error detector. A second, graphical explanation of the N-M\&M's behaviour is presented in the next section.

\section{Graphical Justification for the N-M\&M}

In addition to the theoretical motivation presented in the previous section, a graphical justification for the detectors can also be found. In this section we present such graphical evidence for the case of the N-M\&M. While a similar undertaking can be imparted upon regarding the N-GDD, it can be shown that the plots in that case would be multi-dimensional and hence not particularly conducive to presentation using this medium.

For simplicity we assume a BPSK signal. The desired graphical insight is obtained when one looks as greyscalecoded graphs of the value of the N-M\&M and M\&M,

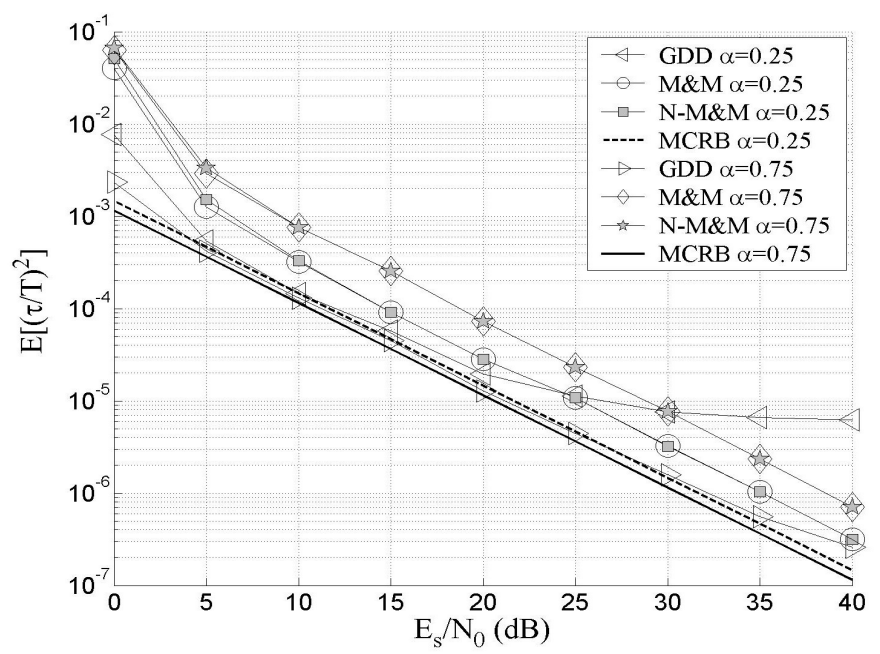

Fig. 7. Normalized timing error variance, BPSK, for $\mathrm{N}$ $\mathrm{M} \& \mathrm{M}, \mathrm{M} \& \mathrm{M}$, and GDD, with $B_{L} T=5 \cdot 10^{-3}$.

upon which received signal points are plotted. This is shown in Fig. 8. Superimposed upon those graphs are identical received signals, but the latter are processed under two different in AGC conditions, the first in which $K=2$, and the second in which $K=7$. As expected, the average output of the N-M\&M detector under both conditions is the same, whilst, if one were to use the M\&M, the average error signal scales with $K$. Intuitive understanding of this phenomenon is attained when one makes note of the fact that the value of $e_{I}(n)$ has radial symmetry vs. the origin $(I(n), I(n-1))=(0,0)$ (as seen in the bottom part of Fig. 8) and the values are bounded by the maximum absolute value of $\left|e_{I}(n)\right| \leq 1$. By contrast, a short glance is sufficient in order to understand the M\&M's inferiority, since the top part of Fig. 8 shows that no radial symmetry exists for the M\&M detector's value, and the latter is unbounded. Thus, with increasing $K$, the signal points fall on progressively darker greyscales, causing the anticipated difference in the M\&M's expected value.

\section{Hardware Implementation}

A compact hardware implementation for the new detectors is easy to discern, and is similar in principle to that found for the detector in [9]. Dealing first with the GDD, the implementation for $v_{I}(n)$ is shown in Fig. 9. The implementation for $v_{Q}(n)$ is analogous, and summation of $v_{I}(n)$ and $v_{Q}(n)$ produces $v(n)$. Since $\left|\left(\operatorname{sign}\left(I_{e}(n+1)\right) I_{o}(n)\right) / \sqrt{I_{e}^{2}(n+1)+I_{o}^{2}(n)}\right|$ $=\left|\operatorname{sign}\left(I_{e}(n+1)\right) \sin \left(\varphi_{n}^{+}\right)\right| \leq 1$ and 
$\left|\left(\operatorname{sign}\left(I_{e}(n)\right) I_{o}(n)\right) / \sqrt{I_{e}^{2}(n)+I_{o}^{2}(n)}\right|$

$=\left|\operatorname{sign}\left(I_{e}(n)\right) \sin \left(\varphi_{n}^{-}\right)\right| \leq 1$ then it is easy to see that the lookup tables in Fig. 9 need only accommodate values in the range $[-1,1]$, consequently allowing them to be feasibly implemented in fixed-point hardware.

The N-M\&M also has an efficient fixed-point hardware implementation through the use of a lookup table. The implementation for $e_{I}(n)$ is shown in Fig. 10. The implementation for $e_{Q}(n)$ is analogously synthesized from the $Q$ channel samples. Summation of $e_{I}(n)$ and $e_{Q}(n)$ produces $e(n)$. Hence summation is the only mathematical operation required. The N-M\&M's independence from $K$ has already been established in the previous sections, and in Section 8 we saw graphical proof that we always have $\left|e_{I}(n)\right| \leq 1$. Thus the lookup table in Fig. 10 (as well as the subsequent datapath) needs only to handle data values in the interval $[-1,1]$, making the implementation quite practical in fixed-point hardware. The same reasoning applies to the $Q$ channel as well.

While implementation of the N-GDD or N-M\&M is somewhat more complicated than that of the original detectors, the advantages gained (such as the ability to relax the requirements upon the AGC and the reduction in the dynamic range that the loop filter needs to handle) may well provide for a reduction in the overall complexity of the receiver, despite the more elaborate TED structure.

\section{Extension to $M>4$}

For the general case of M-PSK, the baseband signal is defined $\quad$ as $\quad m(t) \triangleq \sum_{r=-\infty}^{\infty} \exp \left(j \phi_{r}\right) p(t-r T) \quad$ with $\phi_{r}=2 \pi \cdot m_{r} / M+\pi / M, \quad m_{r} \in\{0,1, \ldots, M-1\}$. The timing detectors discussed in this paper can also be used to synchronize M-PSK symbol streams for $M>4$. In that case[6], in all the preceding equations the $\operatorname{sign}(\bullet)$ operator needs to be replaced with the $\operatorname{Dec}(\bullet)$ (the "Decision") operator, where $\operatorname{Dec}(I(n))+j \cdot \operatorname{Dec}(Q(n))$ is the constellation symbol whose phase is closest to that of the received complex sample $I(n)+j \cdot Q(n)$ (an identical definition applies to $\left.I_{e}(n)+j \cdot Q_{e}(n)\right)$.

For $M>4$, simulations show the following:

- The S-curves of the N-GDD and N-M\&M show a resemblance to those of the GDD and M\&M detectors,

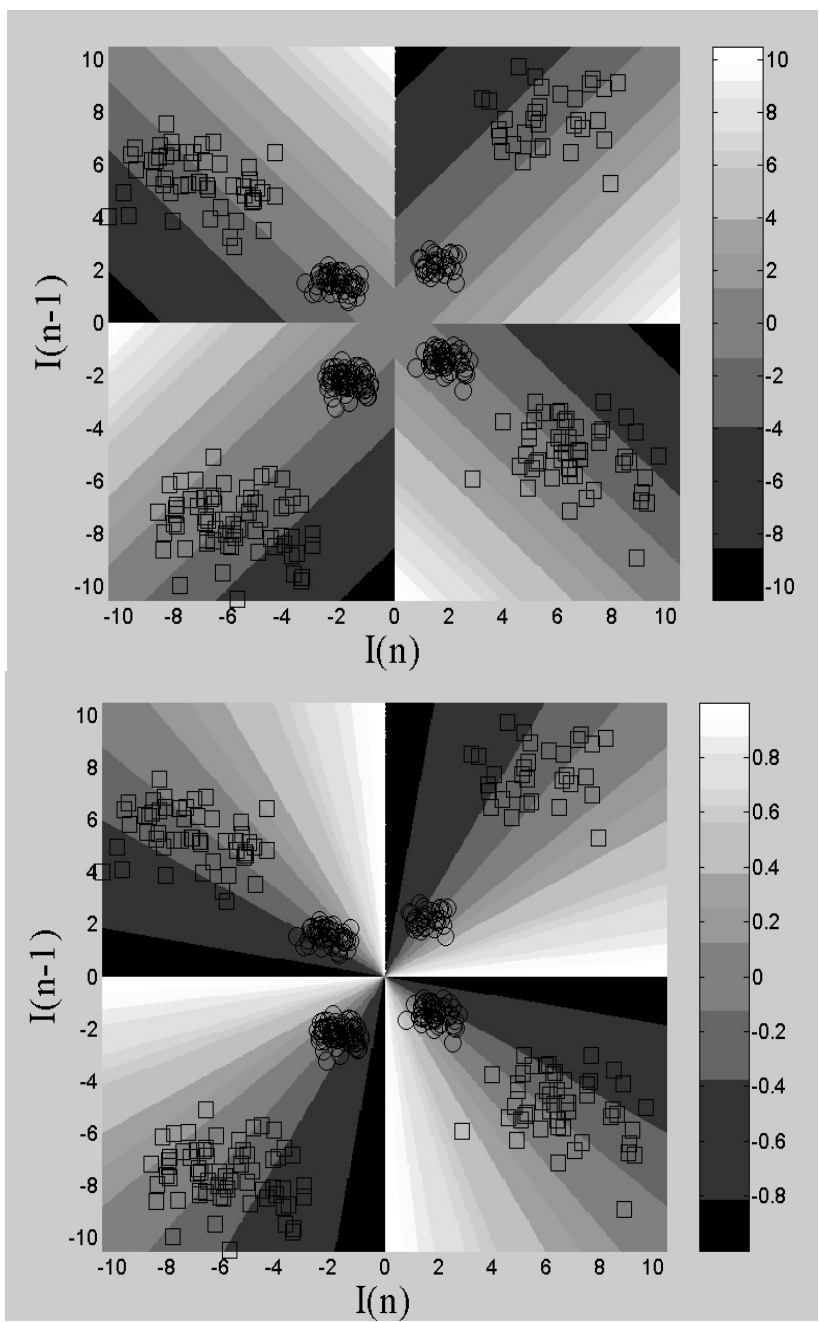

Fig. 8. Greyscale-coded maps of the value of the M\&M detector (top) and the N-M\&M (bottom) upon which two copies of the same received BPSK signal are superimposed: circles are for receivers where $K=2$, and squares are with $K=7 . E_{S} / N_{0}=15 \mathrm{~dB}, \alpha=0.5$, and the timing offset is $\tau=0.2 / T$. The sample signal contains 200 symbols. The average detector output for the N-M\&M is -0.1945 for both values of $K$. For the $\mathrm{M} \& \mathrm{M}$, the average detector output is -0.5179 for $K=2$ and -1.8128 for $K=7$ (or exactly $7 / 2$ times the value with $K=2$, as expected).

similar to the congruence seen for BPSK and QPSK (see Fig. 2-Fig. 4).

- The variance performance results for the GNDA and GDD are only slightly changed from the results given in Fig. 6. The results for the N-M\&M and M\&M are only slightly changed from the results given in Fig. 7. 
- The high-SNR variance performance of the N-GDD is noticeably degraded for $M=8,16$ as compared to BPSK and QPSK. Results for $M=8$ are shown in Fig. 11 (the results for $M=16$ are very similar). However, that performance of the N-GDD is still quite acceptable for use in symbol synchronization circuits.

For $M>4$, the proposed hardware structure for the detectors remains almost unchanged. The only differences are (a) replacement of the $\operatorname{sign}(\bullet)$ operator by the $\operatorname{Dec}(\bullet)$ operator in the lookup tables, and (b) the $\operatorname{Dec}(\bullet)$ data that is needed would be supplied to the lookup tables as part of their input address; this is because the decisions on the $I$ and $Q$ channels are co-related and must be determined jointly. Since $|\operatorname{Dec}(\bullet)| \leq 1$ the dynamic-range advantages outlined earlier still apply to $M>4$, and the detectors remain AGC independent.

\section{Conclusions}

In this paper we presented two new timing error detectors for M-PSK receivers. The detectors are normalized modifications of the Gardner and Mueller \& Müller Decision Directed detectors. The new detectors were characterized using stochastic analysis, and were found to have similar phase error variance performance as the original detectors while providing increased resilience to fading and AGC effects. Furthermore, a hardware implementation structure for the new detectors was suggested, which allows them to be implemented in an FPGA or ASIC using relatively little logic resources.

\section{References}

[1] K. Mueller and M. Müller, "Timing recovery in digital synchronous data receivers," IEEE Trans. Commun., vol. 24, no.5, p. 516- 53, May 1976.

[2] F. M. Gardner, "A BPSK/QPSK timing error detector for sampled receivers," IEEE Trans. Commun., vol. COM-34, no. 5, pp. 423-429, May 1986.

[3] U. Mengali and A. N. D'Andrea, Synchronization Techniques for Digital Receivers. New York: Plenum Press, 1997.

[4] J. D. Gibson (editor), The Communications Handbook, $2^{\text {nd }}$ ed. Boca Raton, FL: CRC Press, 2002.

[5] A. Blanchard, Phase-Locked Loops Application to Coherent Receiver Design. New York: John Wiley \& Sons, 1976.

[6] W. G. Cowley and L. P. Sabel, "The performance of two symbol timing recovery algorithms for PSK demodulators," IEEE Trans. Commun., vol. 42, no. 6, pp. 2345-2355, Jun. 1994.

[7] M. Moeneclaey and T. Batselé, "Carrier-independent NDA symbol synchronization for M-PSK, operating at only one sample per symbol," Proc. GLOBECOM '90, San Diego, CA, vol. 1, p. 594-598.

[8] D. Verdin and T. C. Tozer, "Symbol-timing recovery for M-PSK modulation schemes using the signum function,"

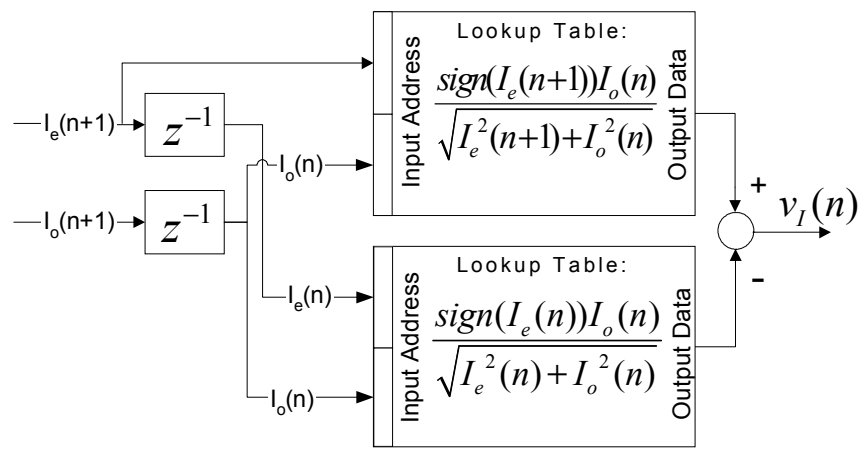

Fig. 9. Hardware implementation of $v_{I}(n)$. The $z^{-1}$ blocks are registers clocked at a rate of $1 / T$.

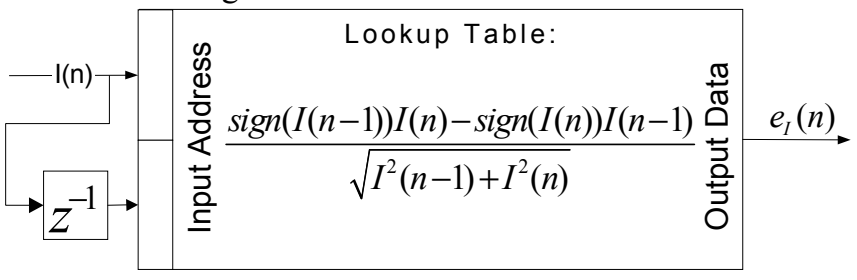

Fig. 10. Hardware implementation for $e_{I}(n)$. The $z^{-1}$ block is a register clocked at a rate of $1 / T$.

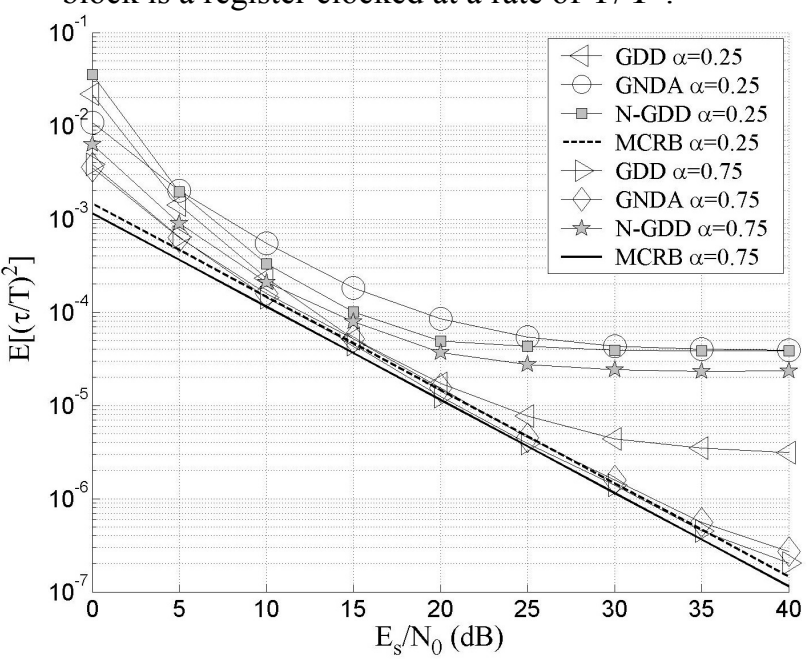

Fig. 11. Normalized timing error variance, 8 -PSK, for $\mathrm{N}$ GDD, GDD, and GNDA, with $B_{L} T=5 \cdot 10^{-3}$.

IEE Colloquium on New Synchronisation Techniques for Radio Systems, 27 Nov 1995, p. 2/1 -2/7.

[9] Y. Linn, "A new NDA timing error detector for BPSK and QPSK with an efficient hardware implementation for ASIC-based and FPGA-based wireless receivers," Proc. ISCAS 2004, Vancouver, BC, Canada, May 23-26, 2004, vol. 4, pp. 465-468.

[10] J. G. Proakis, Digital Communications, $4^{\text {th }}$ ed., New York: McGraw-Hill, 2001.

[11] D. Verdin and T. C. Tozer, "Symbol timing recovery scheme tolerant to carrier phase error," IEE Electronic Letters, vol. 30, no. 2, pp. 116-117, Jan. 1994. 